\title{
TBX21 predicts prognosis of patients and drives cancer stem cell maintenance via the TBX21-IL-4 pathway in lung adenocarcinoma
}

\author{
Shuangtao Zhao ${ }^{1 \dagger}$, Wenzhi Shen ${ }^{2,3 \dagger}$, Jiangyong Yu ${ }^{1}$ and Luhua Wang ${ }^{1 *}$
}

\begin{abstract}
Background: The Th1 cell-specific transcription factor TBX21 functions as a regulator of expression of a Th1 cytokine, interferon gamma (IFN- $\gamma$ ). However, the specific function of TBX21 correlated with cancer stemness remains unclear.

Methods: Using univariate and multivariate survival analysis, TBX21was identified as an independent predictive factor and was associated with poor prognosis in 1389 patients with lung adenocarcinoma (LUAD). Its mechanism in the prognosis was explored by functional enrichment analysis and validated in bioexperiments.

Results: In the training and test sets, TBX21 could classify 1389 LUAD patients into high and low-risk groups with significantly different prognosis $(P<0.01)$. Its prognostic power was independent of other clinical factors including stage, age, gender and smoking status. Functional studies indicated that downregulating TBX21 in lung cancer cells decreased the fraction of cancer stem cells and their sphere and tumor initiation frequency. Furthermore, the study showed that TBX21 activation transduced a TBX21-IL-4 signaling cascade to promote tumor initiation, tumor growth and expression of stemness markers.

Conclusions: These data demonstrated a key role of TBX21 in the maintenance of cancer stemness and that the TBX21-IL-4 pathway is a crucial factor contributing to lung carcinogenesis.
\end{abstract}

Keywords: Lung adenocarcinoma, TBX21, Prognosis, Cancer stemness, IL-4

\section{Background}

Lung cancer remains the most common cancer in the last decades and is still the primary cause of cancer death worldwide, including China [1]. Nonsmall cell lung cancer (NSCLC) accounts for approximately $85 \%$ of lung cancers [2] and lung adenocarcinoma (LUAD) is currently the predominant histological subtype of NSCLC. Although recent advances in multimodality therapy improve the clinical outcome [3], patients with LUAD still have a high rate of relapse $(30-66 \%)[4,5]$ and will die because of cancer

\footnotetext{
* Correspondence: wlhwq@yahoo.com

${ }^{\dagger}$ Equal contributors

'Department of Radiation Oncology, National Cancer Center/Cancer Hospital, Chinese Academy of Medical Sciences and Peking Union Medical College, Beijing 100021, China

Full list of author information is available at the end of the article
}

recurrence [5, 6]. Despite morphological assessment for stratifying patients into different risk groups, it is clear that the overall survival (OS) rates were still $13-58.3 \%$ at 5 years for LUAD patients with high invasiveness and early metastasis [2]. Therefore, it is urgent to develop novel molecular prognosis biomarkers for predicting the prognosis and identifying the high-risk subgroup of LUAD patients with early stage who might benefit from comprehensive therapy.

Previous studies reported that stemness of cancer stem cells (CSCs) may enable CSCs to metastasize and regrow tumors [7], and can be acquired by nonstem cancer cells as they dedifferentiate in response to multiple stimuli $[8,9]$ including inflammatory response. The inflammatory microenvironment in the tumor is activated to drive tumor development as the seventh hallmark of cancer [8]. Li et al. [10] reported that the inflammation-related gene $I L-1 \beta$ 
promotes stemness and invasiveness of colon tumor through activation of CSC self-renewal and EMT. Zhao et al. [11] reported that a three inflammatory gene model including $I L-6, I L-1 A$ and CSF3 could predict survival of diffuse large B-cell lymphoma patients, and patients with high-risk score of this signature had significantly shorter survival than those with low-risk score. The pathway analysis comprised of IRGs could determine genetic risk factors for cancers that might have an underappreciated modest inflammatory component.

TBX21 is a member of a phylogenetically conserved family of genes and Tbx 21 protein is a Th1 cell-specific transcription factor that controls expression of the hallmark Th1 cytokine, interferon gamma (IFN- $\gamma$ ) $[12,13]$. Recently, an increased incidence of TBX21 has been linked to cancer development [14, 15]. However, the specific function of TBX21 associated with cancer stemness or the details remains unclear. In this study, to develop a reliable prognostic model identifying LUAD patients with high-risk status, we screened the significant gene TBX21 from 1027 IRGs, constructed a model to predict survival and further validated it in GEO datasets. Then we investigated the potential biological function of TBX21 in disease progression with biological experiment and confirmed that TBX21 promoted the cancer stemness of tumor cells to reduce the survival time of patients with LUAD.

\section{Methods}

\section{Patient datasets}

The clinical data and gene expression profiles of LUAD patients and normal controls were downloaded from the GEO datasets (https://www.ncbi.nlm.nih.gov/gds) and The Cancer Genome Atlas (TCGA) (https://cancergenome.nih.gov). After removal of LUAD patients with missing values, a total of 1421 LUAD patients and 51 normal controls were analyzed in this study, including 116 patients form GSE50081, 159 patients from GSE31210, 71 patients from GSE30219, 302 patients from GSE72094, 399 patients from TCGA, 342 patients and 19 normal controls from GSE68465, and 32 patients and 32 normal controls from GSE32863. Detailed clinical features of patients from the training data and validated data are presented in Table 1.

\section{Statistical analysis}

Cutoff values were established based on the median expression of the associated genes in each dataset. Pearson's chisquare test analysis of variance was used to analyze statistical significance in demographic and clinical characteristics [16]. The $t$ test was used to compare the distributive difference of the associated gene expression between patients and normal controls, or between the high-risk score group and the low-risk score group. The correlation between TBX21 and IL-4 expression was calculated with
Spearman correlation analysis in the integrated GEO dataset $(n=1389)$. The association between TBX21 expression value and OS of patients was evaluated by univariate Cox proportional hazards regression analysis. Survival differences between low and high-risk groups in each dataset were assessed by Kaplan-Meier estimation, and compared by log-rank test [17]. Multivariate Cox proportional hazards regression analysis and data stratification analysis were performed in our study to explore whether the predictive power of TBX21 was independent of the other clinical factors. Hazard ratios (HRs) and 95\% confidence intervals (CIs) were calculated in each dataset. The prognostic performance at 3,5 or 10 years was measured by timedependent ROC curves. Statistical analysis was conducted with SPSS 13.0, and presented with R3.2.5 software. Results were considered statistically significant at $P<0.05$.

\section{Functional enrichment analysis}

DAVID Bioinformatics Tool was utilized to perform the functional enrichment of target genes. The biological processes associated with protein-coding genes were identified by conducting gene ontology analysis with this tool. In our study, $P<0.05$ was defined as a significant threshold. The pathways involved in the targeted gene were predicted by functional enrichment analysis of KEGG in DAVID Bioinformatics Resources 6.8 (https://david.ncifcrf.gov). Finally, the Enrichment Map plugin for Cytoscape was applied to visualize the biological process organization.

\section{Cell culture}

Human lung cancer cell line A549 was purchased from ATCC and was recently authenticated by cellular morphology and short tandem repeat analysis at Microread Inc. (Beijing, China; May 2014) according to the guideline from ATCC. A549 cells were infected with lentivirus carrying pLV-H1-shRNA-puro or pLV-EF1 $\alpha$-TBX21-puro plasmid, followed by selection using $2 \mu \mathrm{g} / \mathrm{ml}$ puromycin to generate polyclonal cell populations.

\section{Immunohistochemistry}

Immunostaining was performed on paraffin human lung cancer tissue slices. Expression levels of TBX21 in the slices were scored according to the percentage of TBX21-positive cells in each lung tissue. The images were recorded by Olympus BX51 Epi-fluorescent microscopy under a $10 \times$ or $40 \times$ objective (Olympus Co., Tokyo, Japan) [18].

\section{Western blotting}

Cell lysates from different cell lines were prepared with RIPA buffer in the presence of protease inhibitor cocktails and Phosphatase Inhibitor Cocktail 2 and 3 (P8340, P5726 and P0044; Sigma-Aldrich, St Louis, MO, USA). Protein $(20-50 \mu \mathrm{g})$ was separated by $8-15 \%$ Tris- 
Table 1 Correlation between TBX21 expression and different clinical characteristics in LUAD patients enrolled in the study

\begin{tabular}{|c|c|c|c|}
\hline Variable & Low TBX21 expression group & HighTBX21 expression group & $P$ value $^{\#}$ \\
\hline \multicolumn{4}{|l|}{ GSE50081 } \\
\hline Stage & & & 0.385 \\
\hline । & $37(78.7 \%)$ & $45(71.4 \%)$ & \\
\hline$\geq \|$ & $10(21.3 \%)$ & $18(28.6 \%)$ & \\
\hline Age & & & 0.245 \\
\hline$<65$ years & $14(26.4 \%)$ & $23(36.5 \%)$ & \\
\hline$\geq 65$ years & $39(73.6 \%)$ & $40(63.5 \%)$ & \\
\hline Gender & & & 0.877 \\
\hline Female & $26(49.1 \%)$ & $30(47.6 \%)$ & \\
\hline Male & $27(50.9 \%)$ & $33(52.4 \%)$ & \\
\hline Smoking & & & 0.364 \\
\hline No & $11(20.8 \%)$ & $9(14.3 \%)$ & \\
\hline Yes & $35(66.0 \%)$ & $49(77.8 \%)$ & \\
\hline NA & $7(13.2 \%)$ & $5(7.9 \%)$ & \\
\hline \multicolumn{4}{|l|}{ GSE31210 } \\
\hline Stage & & & 0.296 \\
\hline । & $74(78.7 \%)$ & $45(71.4 \%)$ & \\
\hline$\geq \|$ & $20(21.3 \%)$ & $18(28.6 \%)$ & \\
\hline Age & & & 0.107 \\
\hline$<65$ years & $71(74.0 \%)$ & $39(61.9 \%)$ & \\
\hline$\geq 65$ years & $25(26.0 \%)$ & $24(38.1 \%)$ & \\
\hline Gender & & & 0.382 \\
\hline Female & $51(53.1 \%)$ & $29(46.0 \%)$ & \\
\hline Male & $45(46.9 \%)$ & $34(54.0 \%)$ & \\
\hline Smoking & & & 0.419 \\
\hline No & $52(54.2 \%)$ & $30(47.6 \%)$ & \\
\hline Yes & 44 (45.8\%) & 33 (52.4\%) & \\
\hline \multicolumn{4}{|l|}{ GSE30219 } \\
\hline Stage & & & 0.801 \\
\hline I & 27 (87.1\%) & 34 (85\%) & \\
\hline$\geq \|$ & 4 (12.9\%) & $6(15 \%)$ & \\
\hline Age & & & 0.583 \\
\hline$<65$ years & 19 (73.1\%) & $26(66.7 \%)$ & \\
\hline$\geq 65$ years & 7 (26.9\%) & $13(33.3 \%)$ & \\
\hline Gender & & & 0.376 \\
\hline Female & 9 (29.0\%) & 8 (20.0\%) & \\
\hline Male & $22(71.0 \%)$ & 32 (80.0\%) & \\
\hline \multicolumn{4}{|l|}{ GSE68465 } \\
\hline Stage & & & 0.448 \\
\hline I & 62 (36.3\%) & 55 (32.4\%) & \\
\hline$\geq \|$ & 109 (63.7\%) & 115 (67.6\%) & \\
\hline Age & & & 0.746 \\
\hline$<65$ years & 85 (49.7\%) & $82(48.0 \%)$ & \\
\hline$\geq 65$ years & $86(50.3 \%)$ & 89 (52.0\%) & \\
\hline
\end{tabular}


Table 1 Correlation between TBX21 expression and different clinical characteristics in LUAD patients enrolled in the study (Continued)

\begin{tabular}{|c|c|c|c|}
\hline Variable & Low TBX21 expression group & HighTBX21 expression group & $P$ value \\
\hline Gender & & & 0.175 \\
\hline Female & $81(47.4 \%)$ & $93(54.7 \%)$ & \\
\hline Male & $90(52.6 \%)$ & 77 (45.3\%) & \\
\hline Smoking & & & 0.105 \\
\hline No & 19 (11.1\%) & 22 (12.9\%) & \\
\hline Yes & $108(63.2 \%)$ & $121(70.8 \%)$ & \\
\hline NA & $44(25.7 \%)$ & $28(16.3 \%)$ & \\
\hline \multicolumn{4}{|l|}{ GSE72094 } \\
\hline Stage & & & 0.570 \\
\hline I & 99 (63.5\%) & $88(66.7 \%)$ & \\
\hline$\geq \|$ & 57 (36.5\%) & 44 (33.3\%) & \\
\hline Age & & & 0.207 \\
\hline$<65$ years & $51(30.0 \%)$ & 31 (23.5\%) & \\
\hline$\geq 65$ years & 119 (70.0\%) & $101(76.5 \%)$ & \\
\hline Gender & & & 0.955 \\
\hline Female & $92(54.1 \%)$ & $71(53.8 \%)$ & \\
\hline Male & 78 (45.9\%) & $61(46.2 \%)$ & \\
\hline Smoking & & & 0.103 \\
\hline No & $21(12.4 \%)$ & 7 (5.3\%) & \\
\hline Yes & $122(71.8 \%)$ & $100(75.8 \%)$ & \\
\hline NA & 27 (15.8\%) & 25 (18.9\%) & \\
\hline \multicolumn{4}{|l|}{ TCGA LUAD } \\
\hline Stage & & & 0.805 \\
\hline I & $114(59.1 \%)$ & $118(57.8 \%)$ & \\
\hline$\geq \|$ & 79 (40.9\%) & $86(42.2 \%)$ & \\
\hline Age & & & $0.022^{*}$ \\
\hline$<65$ years & $95(47.5 \%)$ & $7(5.3 \%)$ & \\
\hline$\geq 65$ years & $98(49.0 \%)$ & $100(75.8 \%)$ & \\
\hline NA & 27 (15.8\%) & 25 (18.9\%) & \\
\hline Gender & & & 0.337 \\
\hline Female & $104(52.0 \%)$ & $113(56.8 \%)$ & \\
\hline Male & 96 (48.0\%) & 86 (43.2\%) & \\
\hline Smoking & & & 0.056 \\
\hline No & 71 (35.5\%) & $53(26.6 \%)$ & \\
\hline Yes & $129(64.5 \%)$ & $146(73.4 \%)$ & \\
\hline
\end{tabular}

LUAD lung adenocarcinoma, TCGA The Cancer Genome Atlas, NA not available \#Pearson's chi-square test

*Fisher's exact test

acrylamide gels and transferred onto PVDF membrane The membrane was blocked in 5\% skim milk, subsequently incubated with primary antibodies at $4{ }^{\circ} \mathrm{C}$ overnight followed by incubation with peroxidase-conjugated goat anti-mouse IgG or goat anti-rabbit IgG and developed with Pierce ECL reagent (catalog \#17153; Millipore, Billerica, MA, USA) [19].
Flow cytometry

We stained $1 \times 10^{6}$ tumor cells $/ \mathrm{ml}$ with either $5 \mu \mathrm{g} / \mathrm{ml}$ HOECHST 33342 dye or HOECHST dye plus $100 \mathrm{mM}$ Verapamil hydrochloride, to block dye efflux, at $37^{\circ} \mathrm{C}$ for $30 \mathrm{~min}$, as described previously (http://www.bdbioscien ces.com). At the end of the staining period, cells were resuspended in cold staining buffer containing $2 \mu \mathrm{g} / \mathrm{ml}$ 
propidium iodide (PI) for dead cell discrimination. The HOECHST dye was excited at $350 \mathrm{~nm}$, and its fluorescence measured at two wavelengths $(450 / 20 \mathrm{~nm}$ bandpass filter and 675LP optical filter).

\section{Mammosphere formation assay}

Cells were collected and rinsed to remove serum, then dissociated to single-cell suspension in serum-free DMEM/F12 medium supplemented with $100 \mathrm{IU} / \mathrm{ml}$ penicillin, $100 \mu \mathrm{g} / \mathrm{ml}$ streptomycin, $20 \mathrm{ng} / \mathrm{ml}$ human recombinant epidermal growth factor (hREGF), $20 \mathrm{ng} / \mathrm{ml}$ human recombinant basic fibroblast growth factor (bFGF) and 2\% B27 supplement (Invitrogen). Cells were subsequently cultured in ultralow-attachment 24-well plates (Corning Inc.) at a density of no more than 500 cells/well.

\section{Luciferase assay}

To construct the luciferase reporter vectors, the IL-4 gene promoter fragment was amplified from human genomic DNA and cloned into the firefly luciferase plasmid pGL3basic-IRES. For reporter assays, the aforementioned constructs along with pRL-TK plasmid (internal reference) were cotransfected into A549 cells. Cell lysates were then collected and analyzed with the Dual-Luciferase Reporter Assay System (E1910; Promega) following its manual.

\section{Chromatin immunoprecipitation assays}

Chromatin immunoprecipitation (ChIP) assays were performed as described previously (17-371EZ-CHIP, Millipore, Billerica, MA, USA). Briefly, after sonication, $10 \%$ of each sample was saved as total input, $45 \%$ of each sample was incubated with $5 \mu \mathrm{g}$ of anti-TBX21 antibody (catalog \#sc-126; Santa Cruz, TX, USA), and the remaining $45 \%$ of each sample was incubated with $5 \mu \mathrm{g}$ of normal IgG (catalog \#sc-2025; Santa Cruz) at $4{ }^{\circ} \mathrm{C}$ overnight and then with $50 \mu \mathrm{l}$ (bead volume) of Pure Proteome proteinG magnetic beads (catalog \#LSKMAGG02; Millipore, CA, USA) at $4{ }^{\circ} \mathrm{C}$ for $4 \mathrm{~h}$. After washing and reverse crosslinking, DNA was extracted with phenolchloroform, precipitated with ethanol and dissolved in water. The TBX21 binding sites in the IL- 4 gene promoter region were identified by Chip-PCR.

\section{Animal study}

A549-shTBX21 and A549-sc cells $\left(5 \times 10^{6}\right.$ cells $)$ were injected into NOD/SCID mice subcutaneously and tumors were palpable at 12 days after injection. Eighteen days after injection the mice were sacrificed, and tumors were harvested and analyzed. The tumors were then digested with enzyme into single cell suspension, and cell sorting was performed to sort out the side population cells using FACS. Further, the mRNA and proteins were extracted from the sorted SP cells.

\section{Results}

\section{Upregulation of TBX21 was associated with poor} prognosis of patients with lung adenocarcinoma

To investigate IRGs' prognostic role in LUAD, 1027 IRGs (Additional file 1: Table S1) from others' previous research [20] were firstly evaluated in 1389 LUAD patients from six datasets. With the median value as a cutoff point, upregulation of TBX21 was identified as a poor prognostic biomarker in patients with LUAD. The clinical baseline characteristics were not significantly different between the low and high-expression groups of TBX21 (Table 1).

Next, the log-rank test was accomplished between the low and high-value groups in this dataset. We found that patients with high-value expression of TBX21 had significantly higher risk than those with low values $(\mathrm{HR}=$ 2.008, 95\% CI 1.284-3.396, $P=0.003$; Fig. 1a). The 3year OS rates and the 5 -year OS rates of patients in the high-risk group were $69.53 \%$ and $62.58 \%$, respectively, whereas the corresponding rates in the low-risk group were $93.30 \%$ and $78.09 \%$. These results indicated that TBX21 could distinguish LUAD patients with high or low risk of survival.

To assess the reproducibility of the prognostic model, another 1273 patients from five independent datasets (accession numbers GSE31210, GSE30219, GSE68465, GSE72094 and TCGA LUAD) were used to validate its predictive ability. With the same cutoff value derived from the training dataset, LUAD patients in each dataset were then divided into two groups: high and low-risk groups. Similar to the earlier result, the significantly different survival between the two predictive groups $(P<$ 0.01; Fig. 1b-f) was demonstrated by survival analysis. In GSE31210, the 3-year OS rates and the 5-year OS rates of patients in the high-risk group were less than the corresponding rates in the low-risk group (88.57\% and $74.70 \%$ vs $95.59 \%$ and $92.71 \%$ ), respectively (Fig. 1 b). The LUAD patients with high-risk scores showed significantly shorter survival than those with low-risk scores (median survival 10.60 years vs 16.10 years $(P=0.030)$ in GSE30219, 4.18 years vs 9.50 years $\left(P=2.58 \times 10^{-6}\right)$ in GSE68465, 3.07 years vs 4.27 years $(P=0.012)$ in GSE72094, and 5.95 years vs 13.58 years $\left(P=8.03 \times 10^{-}\right.$ ${ }^{6}$ ) in TCGA LUAD) (Fig. 1c-f). These results demonstrated that the prognostic model of TBX21 was robust to identify LUAD patients with poor survival.

\section{The prognostic power of TBX21 was independent of other clinicopathological factors}

To evaluate whether the prognostic power of TBX21 was independent of other clinical factors, multivariate Cox proportional hazard regression analysis was performed with risk score and other available clinicopathological variables (including patients' age, 

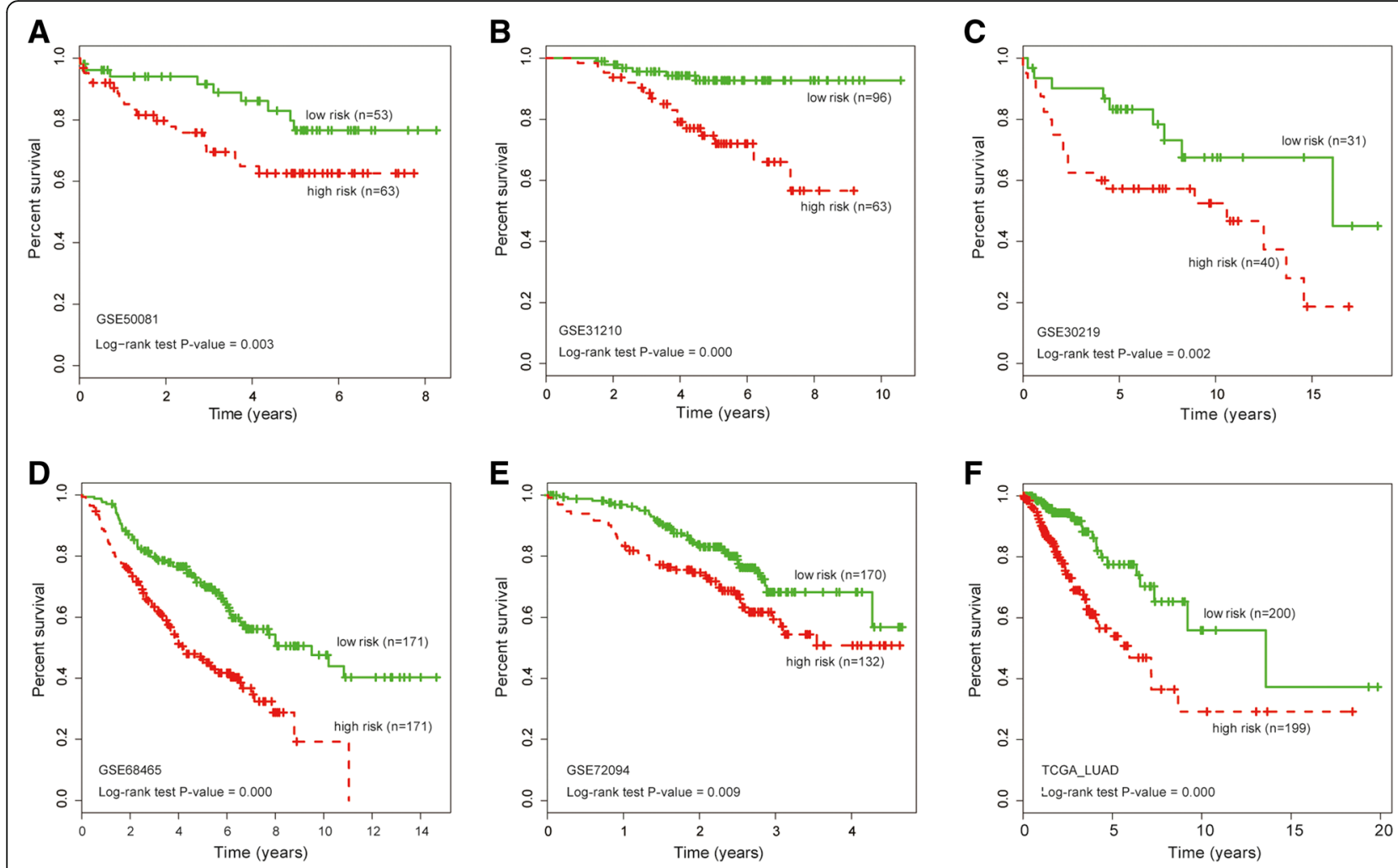

Fig. 1 Prognostic model validating predictive power of TBX21 gene in independent datasets. Kaplan-Meier survival curves between low-risk group and high-risk group in (a) training dataset and ( $\mathbf{b}-\mathbf{f})$ other validated datasets

gender, clinical stage and smoking status) as covariates in these six LUAD datasets (Table 2). The results showed that TBX21 was still significantly correlated with survival when adjusted for age, gender, clinical stage and smoking status in the training dataset $(\mathrm{HR}=1.943,95 \%$ CI $1.234-3.060, P=0.004)$ and in the other five validated datasets $(H R=1.018$, 95\% CI 1.002-1.035, $P=0.028$ for GSE31210; $\mathrm{HR}=$ 2.625, 95\% CI 1.284-5.365, $P=0.008$ for GSE30219; $\mathrm{HR}=1.009,95 \% \mathrm{CI} 1.007-1.012, P=0.000$ for GSE68465; $\mathrm{HR}=1.469,95 \%$ CI 1.127-1.914, $P=$ 0.004 for GSE72094; and HR $=1.059$, 95\% CI 1.008$1.112, P=0.023$ for TCGA LUAD; Table 2). However, we also discovered that clinical stage, age and gender were significant in the multivariate analysis in some datasets besides the predictive gene TBX21.

Next, data stratification analysis was carried out according to clinical stage, age or gender in the integrated GEO datasets. First, all patients from the integrated GEO datasets were stratified into two groups with different stage status (stage I, $n=797$ and stage $\geq \mathrm{II}, n=566$ ). As shown in Additional file 2: Figure S1A, the cutoff value of TBX21 could subdivide patients with stage I into the high-risk group and low-risk group with significant survival time $(\mathrm{HR}=2.876,95 \%$ CI 2.087-3.963, $P=7.02 \times$ $\left.10^{-11}\right)$. The OS rates of patients with high-risk score were $76.93 \%$ and $65.70 \%$ at 3 and 5 years, respectively, which were also significantly lower than those from patients with low-risk score whose corresponding proportions were $93.09 \%$ and $84.94 \%$. For the patients with stage $\geq \mathrm{II}$, we obtained a similar result to patients with stage I $(\mathrm{HR}=2.170,95 \%$ CI $1.663-$ 2.832, $P=9.24 \times 10^{-8}$; Additional file 2: Figure S1B). Analogously, the predictive power of TBX21 was further tested for patients with different age or gender. The patients of each subgroup were subclassified into two groups (high and low-risk groups) with significantly different clinical outcome $(P<$ 0.001). The OS rates of patients with high-risk score were significantly worse than those with low-risk score (at 3 and 5 years, $73.12 \%$ vs $87.23 \%$ and $59.12 \%$ vs $79.60 \%$ in younger group (Additional file 2 : Figure S1C), $72.42 \%$ vs $86.56 \%$ and $58.41 \%$ vs $76.36 \%$ in female group (Additional file 2: Figure S1E); median survival (years) 5.04 vs $10.20(P<0.0001$, Additional file 2: Figure S1D) in older group and 5.55 vs $10.83(P<0.0001$, Additional file 2: Figure S1F) in male group). Generally, these results demonstrated that the TBX21 predictive power for prognosis was independent of other clinicopathological factors for patients with LUAD. 
Table 2 Univariate and multivariate Cox regression analysis for lung adenocarcinoma in validated datasets

\begin{tabular}{|c|c|c|c|c|c|c|}
\hline \multirow[t]{2}{*}{ Variable } & \multicolumn{3}{|c|}{ Univariate analysis } & \multicolumn{3}{|c|}{ Multivariate analysis } \\
\hline & $\mathrm{HR}$ & $95 \% \mathrm{Cl}$ & $P$ value & $\mathrm{HR}$ & $95 \% \mathrm{Cl}$ & $P$ value \\
\hline \multicolumn{7}{|l|}{ GSE31210 ( $n=159)$} \\
\hline Gender (male/female) & 1.484 & $0.651-3.387$ & 0.348 & & & \\
\hline Stage $(\geq \| / I)$ & 3.107 & $1.358-7.110$ & 0.007 & 2.971 & $1.295-6.819$ & 0.010 \\
\hline Age ( $\geq 65 /<65$ years) & 1.854 & $0.813-4.231$ & 0.142 & & & \\
\hline Smoking (yes/No) & 2.101 & $0.907-4.867$ & 0.083 & & & \\
\hline TBX21 (high risk/low risk) & 4.550 & $1.960-10.560$ & 0.000 & 1.018 & $1.002-1.035$ & 0.028 \\
\hline \multicolumn{7}{|l|}{ GSE30219 $(n=71)$} \\
\hline Gender (male/female) & 1.111 & $0.464-2.661$ & 0.813 & & & \\
\hline Stage $(\geq \| / /)$ & 1.669 & $0.878-3.173$ & 0.118 & & & \\
\hline Age ( $\geq 65 /<65$ years) & 2.539 & $1.250-5.158$ & 0.010 & 2.267 & $1.108-4.637$ & 0.025 \\
\hline TBX21 (high risk/low risk) & 2.876 & $1.457-5.677$ & 0.002 & 2.625 & $1.284-5.365$ & 0.008 \\
\hline \multicolumn{7}{|l|}{ GSE68465 $(n=342)$} \\
\hline Gender (male/female) & 1.376 & $1.008-1.878$ & 0.044 & 1.425 & $1.033-1.965$ & 0.031 \\
\hline Stage $(\geq \| / /)$ & 1.713 & $1.362-2.153$ & 0.000 & 1.621 & $1.272-2.066$ & 0.000 \\
\hline Age ( $\geq 65 /<65$ years) & 1.266 & $0.928-1.726$ & 0.137 & & & \\
\hline Smoking (yes/no) & 1.045 & $0.626-1.744$ & 0.866 & & & \\
\hline TBX21 (high risk/low risk) & 2.147 & $1.561-2.952$ & 0.000 & 1.009 & $1.007-1.012$ & 0.000 \\
\hline \multicolumn{7}{|l|}{ GSE72094 $(n=302)$} \\
\hline Gender (male/female) & 1.500 & $0.976-2.305$ & 0.065 & & & \\
\hline Stage $(\geq\|/\|)$ & 2.593 & $1.676-4.011$ & 0.000 & 2.682 & $1.734-4.149$ & 0.000 \\
\hline Age ( $\geq 65 /<65$ years) & 1.485 & $0.882-2.501$ & 0.137 & & & \\
\hline Smoking (yes/no) & 1.966 & $0.714-5.413$ & 0.191 & & & \\
\hline TBX21 (high risk/low risk) & 1.424 & $1.091-1.859$ & 0.009 & 1.469 & $1.127-1.914$ & 0.004 \\
\hline \multicolumn{7}{|l|}{ TCGA LUAD $(n=399)$} \\
\hline Gender (male/female) & 0.943 & $0.601-1.480$ & 0.798 & & & \\
\hline Stage $(\geq\|/\|)$ & 3.079 & $1.920-4.937$ & 0.000 & 2.956 & $1.838-4.754$ & 0.000 \\
\hline Age ( $\geq 65 /<65$ years) & 1.375 & $0.865-2.184$ & 0.178 & & & \\
\hline Smoking (yes/no) & 1.102 & $0.676-1.796$ & 0.698 & & & \\
\hline TBX21 (high risk/low risk) & 2.793 & $1.779-4.384$ & 0.000 & 1.059 & $1.008-1.112$ & 0.023 \\
\hline
\end{tabular}

HR hazard ratio, Cl confidence interval, LUAD lung adenocarcinoma, TCGA The Cancer Genome Atlas

\section{Validation of high expression of TBX21 in LUAD}

To confirm the high expression of TBX21 in LUAD, the TBX21 gene was firstly evaluated between adjacent normal and tumor tissues using a microarray assay from 64 samples in GSE32863 and 361 samples in GSE68465. We found that it was significantly upregulated in LUAD tissues compared with normal tissues $(P<0.01$; Fig. $2 \mathrm{a})$. Then the protein expression of TBX21 was determined using an immunohistochemistry assay in LUAD and normal lung sections from 15 samples (10 LUAD tissues and 5 adjacent normal tissues). The results showed that the expression of TBX21 was significantly higher in LUAD samples than in normal samples $(P<0.0001$; Fig. $2 \mathrm{~b}$, c), which was also in line with the mRNA expression profile earlier. These results demonstrated that an increased expression of TBX21 might be associated with the presence of LUAD.

To determine whether TBX21 was upregulated in tumor cell lines, the expression of TBX21 was measured with quantitative RT-PCR and immunoblot analysis between one normal cell line (MRC-5) and two LUAD cell lines (A549 and H1299), respectively. As a result, we discovered that not only mRNA expression but also protein expression of TBX21 was increased in LUAD cell lines compared with the normal cell line (Fig. 2d). Therefore, these studies suggested that these cell lines could be utilized to validate the activated role of TBX21 in LUAD genesis. 

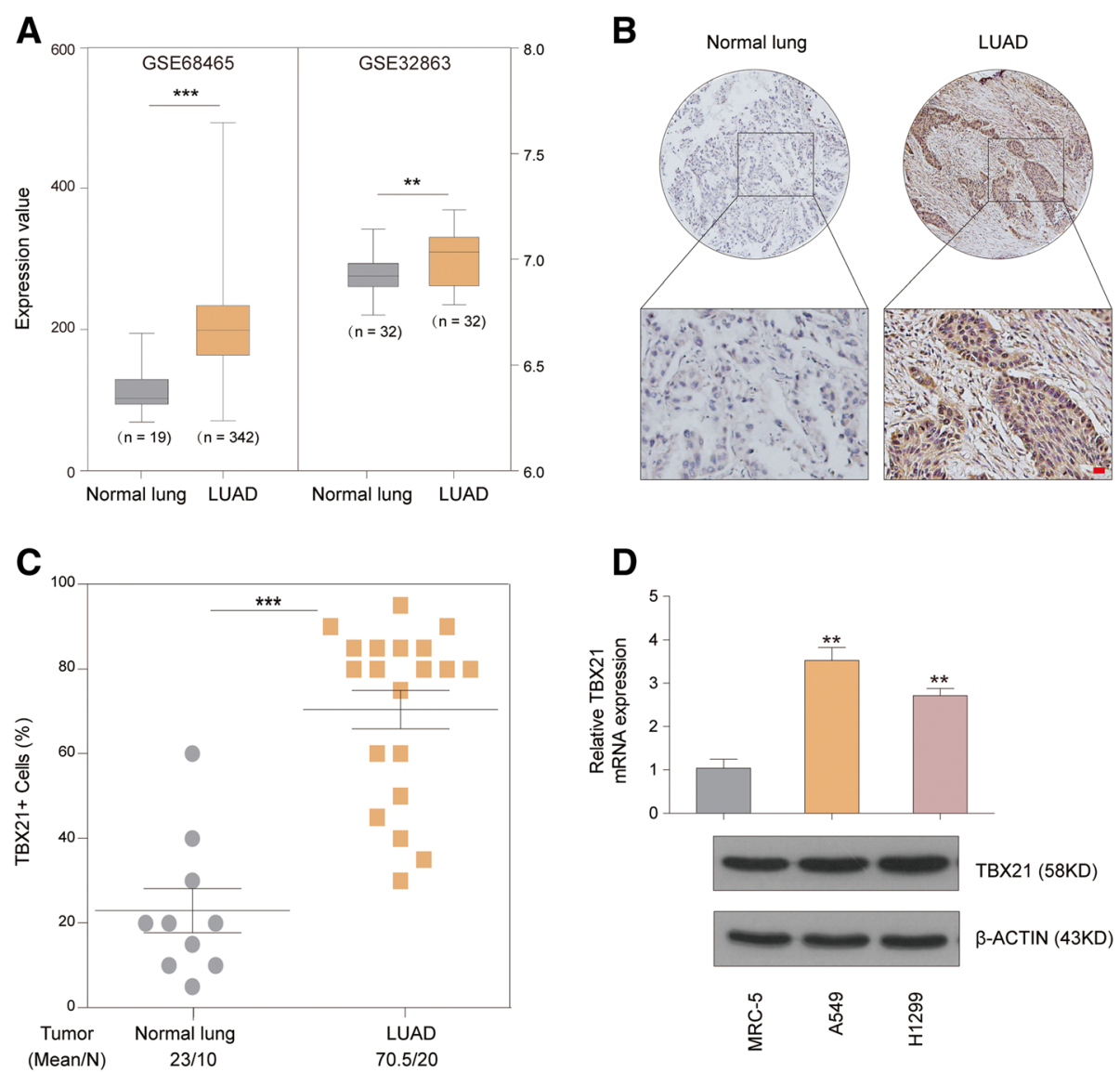

Fig. 2 Validation of expression status of TBX21 between normal samples and LUAD. a mRNA expression of TBX21 is significantly upregulated in LUAD compared with normal lung in GSE32863 and GSE68465 ( ${ }^{* *} P<0.0001$, ${ }^{* *} P<0.01$, $t$-test method). b, c Immunohistochemistry assay shows protein expression of TBX21 is significantly upregulated in LUAD compared with normal lung tissue (*** $<0.001, t$-test method). $\mathbf{d}$ Expression of TBX21 is significantly downregulated in normal cell line (MRC-5) compared with tumor cell lines (A549 and H1299) examined by quantitative RT-PCR method (bar plot, top panel) and immunoblot analysis (bottom panels). Error bars represent SEM (** $P<0.01$, $t$-test method). LUAD lung adenocarcinoma

\section{Validation of stemness-related role of TBX21 in LUAD maintenance}

Previous studies reported that CSC biomarkers such as NANOG [21, 22], OCT4 [21, 23], SOX2 [21, 24], KLF4 $[21,25]$ and ALDH1A1 [21, 26] were associated with a poor prognosis in several cancers. The correlation analysis indicated that the average expression of the five cancer stemness biomarkers was tightly associated with TBX21 in the integrated GEO dataset $(r \geq 0.850, P<$ 0.05; Fig. 3a). Further, we evaluated the expression of these five CSC biomarkers and TBX21 by analyzing the RNA sequence of tumors and the matched nonneoplastic tissue from 520 LUAD patients with diseasefree survival status or overall survival status in the TCGA dataset. The result summarized the overall landscape of the significantly mutated genes in LUAD, and the patients with mutation (amplification, deep deletion, missense mutation and mRNA upregulation) altered in 154 (30\%) of 520 patients (Fig. 3b). Also, we discovered that mRNA upregulation was the dominating mutation form among the five CSC biomarkers and TBX21 (Fig. 3b). We also discovered that the total mean mutation rates of SOX2 and OCT4 $(11 \%$ and $7 \%$, respectively) were higher than the other three CSC biomarkers (3\% for NANOG, $4 \%$ for KLF4 and $4 \%$ for ALDH1A1, respectively) in these patients, which was close to the TBX21 mutation rates (6\%; Fig. 3b). So, we could infer that TBX21 might play a positive role in LUAD maintenance of cancer stemness.

To validate the activated function of TBX21 in mediating cancer stemness in LUAD, the expression of TBX21 was knocked down in A549 cells by stable expression of the control shRNA (sc) or two shRNAs for TBX21. Consistently, the expression of cancer stemness biomarkers, including SOX2 and OCT4, was decreased following the downregulation of TBX21 expression in western blot analyses (Fig. 3c). Then, flow cytometry of TBX21-transfected A549 cells was performed to identify side populations and the result showed that the proportion of side-population (SP) cells was significantly depressed by the loss of TBX21 
A

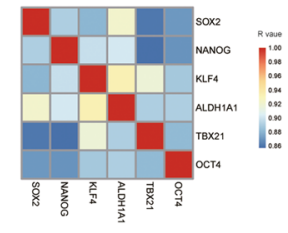

C

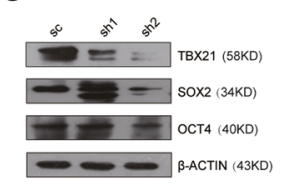

$\mathbf{F}$
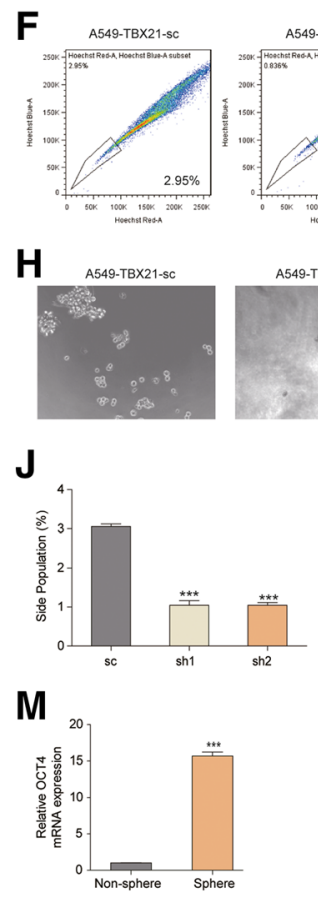

$\mathbf{P}$

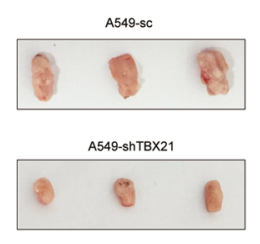

B

B TCGA_LUAD

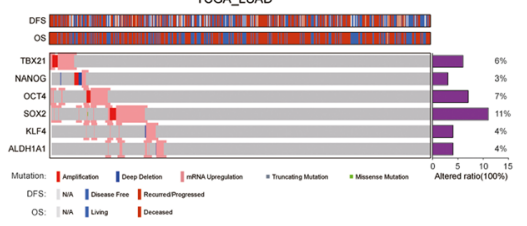

D
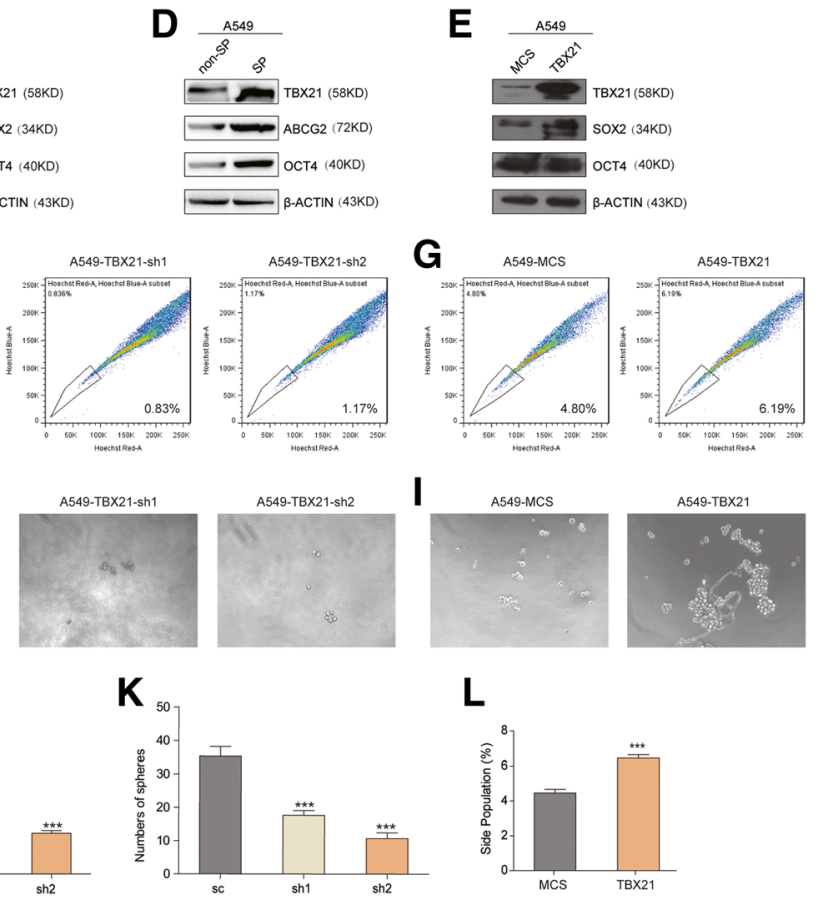

$\mathbf{L}$

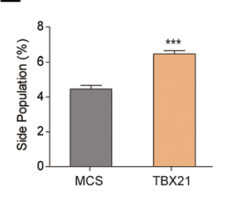

N
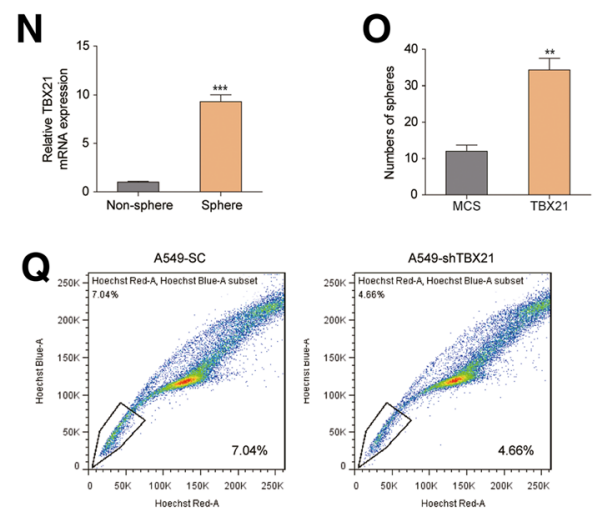

$\mathbf{R}$

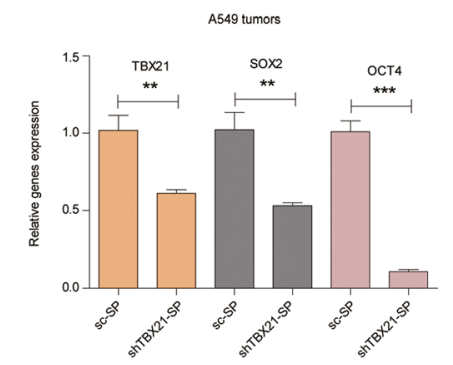

S

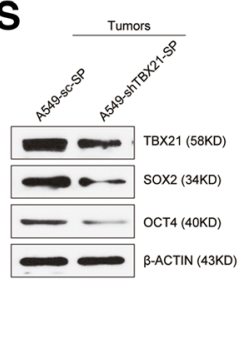

Fig. 3 (See legend on next page.) 


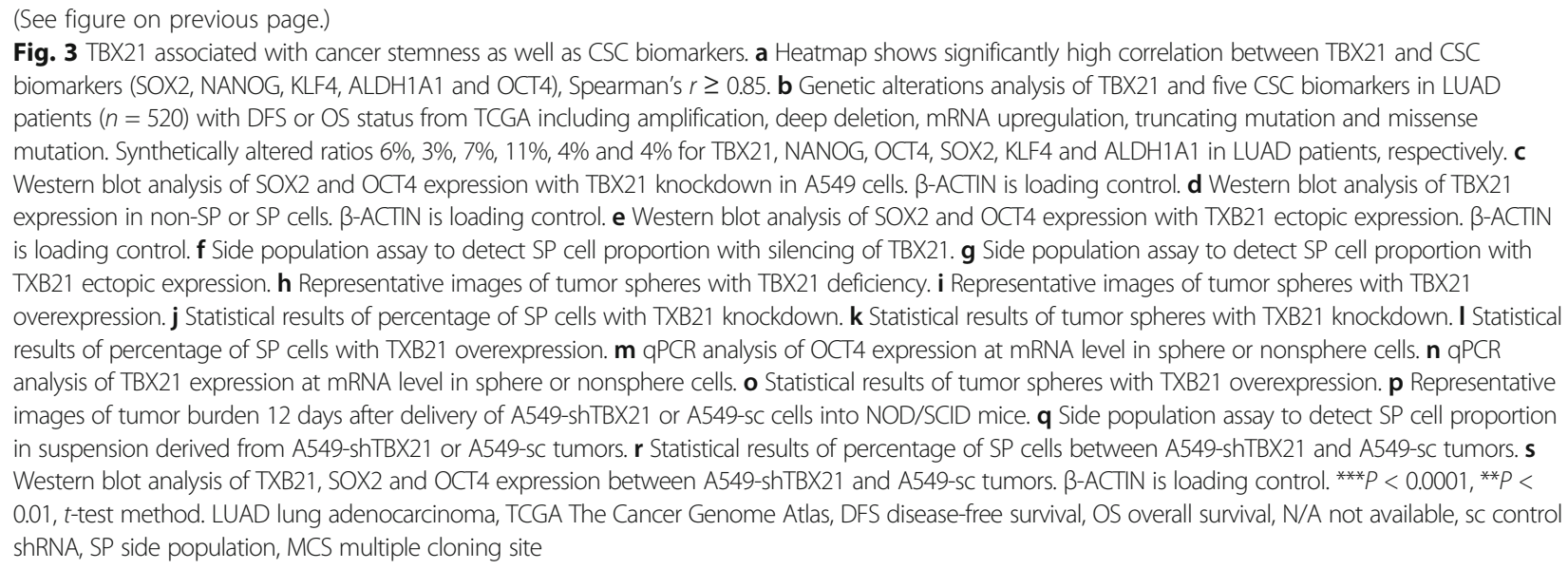

$(P<0.0001$; Fig. 3f, j). As an important feature of cancer stemness, self-renewal of LUAD cells was measured in the sphere formation experiment of A549 cells with sc or shRNAs for TBX21. A549-TBX21-sc cells readily grew spheres in serum-free medium and the presence of TBX21 markedly increased the number and size of spheres compared to that of A549-TBX21-shRNAs (Fig. 3h, k). Moreover, TBX21 expression was measured in SP cells sorted with flow cytometry and evaluated by the protein expression of ABCG2 and OCT4 with western blot assay (Fig. 3d), and in cultured spheres evaluated by mRNA expression of OCT4 with RT-PCR analysis (Fig. 3m). In line with expression of ABCG2 and OCT4, expression of TBX21 was significantly higher in SP cells $(P<0.0001$; Fig. 3d $)$ and tumor spheres $(P<0.0001$; Fig. 3n) compared with non-SP cells or nonspheres. In addition, the TBX21 gene was reconstituted into A549 cells and then increased the protein expression of the stemness markers, including SOX2 and OCT4 (Fig. 3e). In agreement, ectopic expression of TBX21 raised remarkably the proportion of SP cells $(P<0.0001$; Fig. $3 g$, l) and enhanced ability dramatically in the tumor sphere formation of A549 cells $(P<0.0001$; Fig. 3i, o). Taken together, these data indicated that TBX21 promoted the cancer stemness of LUAD cells and played a positive role in maintaining LUAD cell development.

To further identify the role of TBX21 in regulating cancer stemness in vivo, we performed tumor xenograft studies in NOD/SCID mice injected with either A549shTBX21 or A549-sc cells. Similarly, the result showed a marked reduction in tumor growth in the A549shTBX21 group versus the A549-sc control (Fig. 3p). Furthermore, flow cytometry was conducted in the A549-shTBX21 or A549-sc tumors to identify side populations and the result showed that SP cells were significantly depressed by the loss of TBX21 $(P<0.0001$; Fig. $3 q$ ). Then, mRNA and protein expression of TBX21, SOX2 and OCT4 were measured in both SP cells with
RT-PCR analysis and western blot method, respectively. As a result, we discovered that both the mRNA and protein expression of TBX21, SOX2 and OCT4 in SP cells derived from A549-shTBX21 tumor were significantly declined compared with those from A549-sc tumor $(P<$ 0.01 ; Fig. 3r, s). In line with the result in vitro, these data indicated once again that TBX21 could improve the cancer stemness of LUAD cells and positively maintain LUAD development.

\section{IL-4 signaling is indispensable for TBX21 to define cancer stemness}

To identify potential biological processes and pathways involving the TBX21 gene, functional enrichment analysis was performed by GO terms and KEGG pathways between TBX21 and the other protein-coding genes (PCGs). Next, Spearman's correlation coefficient was calculated between TBX21 and the PCGs using paired expression profiles, and the top 20 positively or negatively correlated PCGs were chosen with at least one kind of relationship such as physical interaction, coexpression, predicted, pathway, colocalization, genetic interaction and shared protein domains (Fig. $4 \mathrm{a}$ ). The result revealed that IL-4 associated with TBX21 clustered the most GO functional clusters (including DNA recombination, cell proliferation and activation, immune cell and osteoclast differentiation, and adaptive immune response; Fig. 4a). The average expression of IL- 4 was tightly correlated with TBX21 in 1389 patients with LUAD from the six GEO datasets (Spearman's $r=0.849, P=0.000$; Fig. 4b). As previous studies proved that IL- 4 with high expression value promoted cancer stemness [27] and predicted the poor prognosis [28] in cancers, the results indicated that cancer stemness might depend on the interaction between TBX21 and IL-4 in LUAD cells.

To validate the correlation between TBX21 and IL-4 in cancer stemness regulation, the expression of both genes was detected with RT-PCR. As shown in Fig. 4c, 

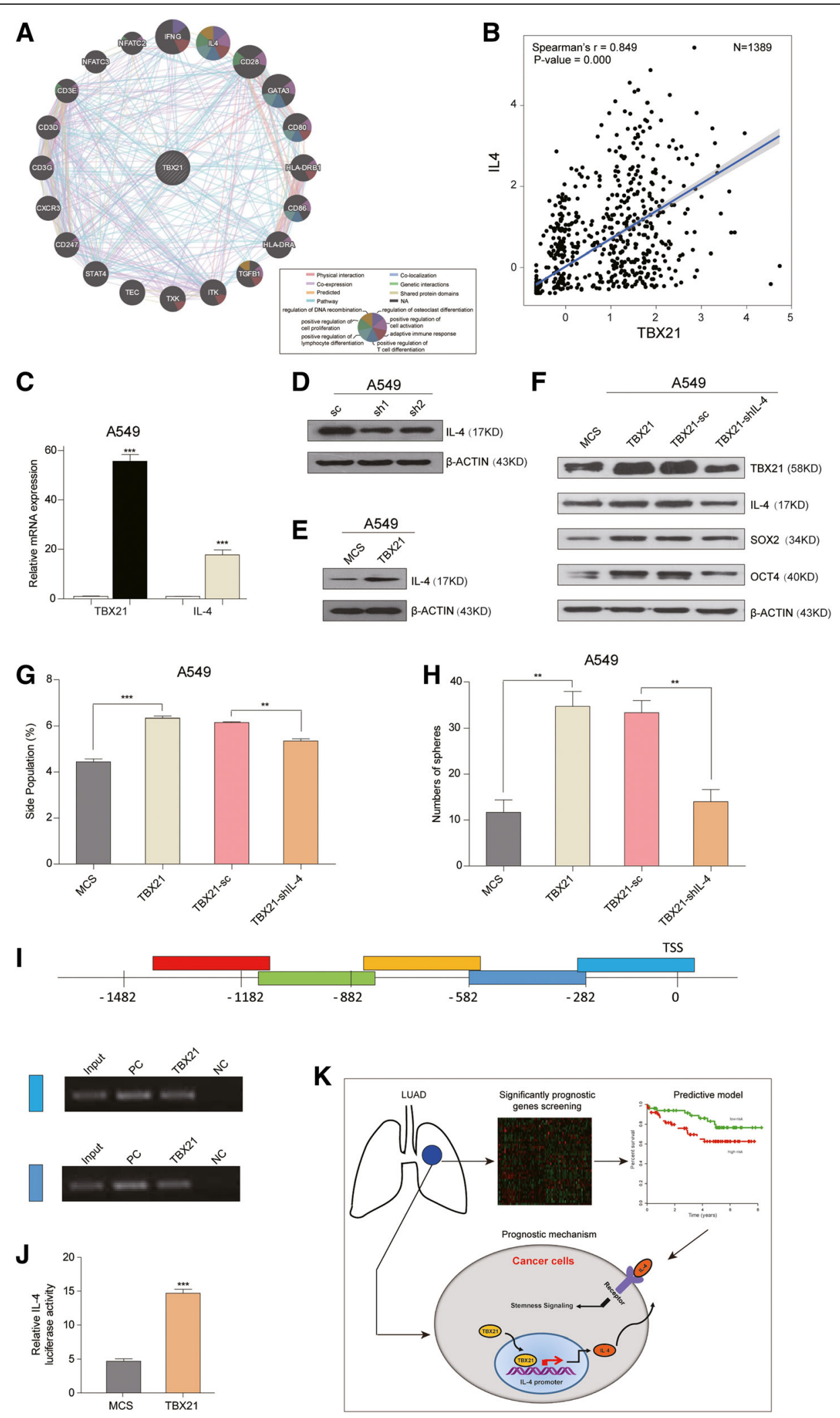

Fig. 4 (See legend on next page.) 


\begin{abstract}
(See figure on previous page.)
Fig. 4 IL-4 is targeted by TBX21 in A549 cells to maintain cancer stemness. a Functional enrichment map for RNA-based expression revealing TBX21 modulated networks. Nodes (circles) with distinct color subparts represent different function enriched in gene sets from DAVID database. Nodes including distinct color subparts have significant overlap with TBX21 predicted targets, and each node connected with TBX21 belongs to the same cluster (MCL cluster algorithm). Distinct color lines between nodes correspond to different interactions among these significant genes (Wilcoxon test $P<0.05$ ). b Correlation of TBX21 and IL-4 mRNA levels (normalized) in integrated dataset. Spearman's correlation and $P$ value indicated. c qPCR analysis of TBX21 and IL-4 expression at mRNA level. d Western blot analysis of IL-4 expression with TXB21 knockdown in A549 cells. $\beta$-ACTIN is loading control. e Western blot analysis of IL-4 expression with TXB21 ectopic expression. $\beta$-ACTIN is loading control. $\mathbf{f}$ Western blot analysis showed IL-4 deficiency rescued SOX2 and OCT4 expression in A549-TBX21 cells. $\beta$-ACTIN is loading control. $\mathbf{g}$ Statistical results of percentage of SP cells with IL-4 knockdown in A549-TBX21 cells. $\mathbf{h}$ Statistical results of tumor spheres with IL-4 knockdown in A549-TBX21 cells. $\mathbf{i}$ CHIP analysis of TBX21-binding sites on IL-4 gene promoter. $\mathbf{j}$ Dual-luciferase assay to detect IL-4 promoter activity after TBX21 overexpression. $\mathbf{k}$ Summary of prognostic model associated with LUAD. Schematic includes two lungs with LUAD, microarray screening, predictive model and mechanism investigation. Blue cell in left lung represents LUAD lesion. Heatmap indicates significant genes correlated with prognosis. ${ }^{* * *} P<$ 0.0001 , ${ }^{*} P<0.01$, $t$-test method. Ellipse demonstrates signaling pathway of TBX21-IL-4 in cancer cells. N/A not available, IL interleukin, sc control shRNA, TSS transcription start site, LUAD lung adenocarcinoma, MCS multiple cloning site, PC positive control, NC negative control
\end{abstract}

mRNA expression of IL-4 was significantly upregulated following the reconstitution of TBX21 expression in A549 cells. Accordingly, the IL-4 expression was detected both in TBX21 deficiency and reconstitution systems with western blot analysis. Obviously, the protein expression of IL-4 was decreased with TBX21 deficiency (Fig. 4d) and increased with TBX21 reconstitution (Fig. 4e). These results suggested that IL-4 might be targeted by TBX21 to mediate cancer stemness.

To prove this conclusion, IL-4 gene was knocked down in the TBX21 reconstitution systems in A549 cells. The result showed that expression of SOX2 and OCT4 was correspondingly reduced accompanied by the loss of IL-4 (Fig. 4f). Furthermore, the SP cells were evaluated with flow cytometry, and the result showed that IL-4 gene deficiency significantly abolished the SP cell increase induced by the TBX21 gene (Fig. 4g). In concordance with the SP, loss of IL-4 expression was discovered to abolish the sphere formation induced by TBX21 (Fig. 4h). Some studies revealed that TBX21 works as a transcription factor and regulates many target genes [14, 29, 30]. The Chip assay was then performed to investigate whether TBX21 could regulate IL-4 gene transcription. The result verified that TBX21 binds to IL-4 gene promoter with a region of approximately 0-600 bp upstream of the transcription start site (TSS) (Fig. 4i). To complement the Chip-PCR assay, IL-4 promoter-derived luciferase reporter construct and TBX21-overexpressed vector were cotransfected into A549 cells. The luciferase assay confirmed that the overexpression of TBX21 really heightened IL-4 promoter activity (Fig. 4j). Taken together, a schematic model correlated with survival of LUAD patients was displayed focusing on the relationship between the TBX21-IL-4 signaling pathway and cancer stemness. TBX21 works as a transcription factor targeting the promoter region of IL-4 which promotes IL-4 expression and secretion. The secreted IL- 4 bound to its receptor on the cancer cell membrane further induced downstream signaling activation to mediate cancer stemness (Fig. 4k). All of the presented results prove that cancer stemness maintenance was governed by the TBX21-IL-4 pathway in LUAD cells.

\section{Discussion}

LUAD is the most common kind of NSCLC [31]. LUAD patients were treated with surgical resection for early stage disease and adjuvant chemotherapy for stage IB or II. Although oncologists usually made the treatment decisions for adjuvant chemotherapy according to the traditional clinical factors such as age, gender, tumor stage, tumor size and so on, LUAD patients still suffered from the high risk of cancer recurrence. A recent study focusing on the molecular mechanisms of LUAD showed that LUAD was a heterogeneous cancer including diverse morphologic and molecular features [32]. The response to adjuvant chemotherapy has been proven to correlate with the heterogeneous molecular features [33]. So, it is urgent to develop some new molecular biomarkers to further stratify LUAD patients for identifying cancers with a high-risk value who will benefit from the adjuvant chemotherapy and patients with a low-risk value who will be able to avoid overtreatment.

In the past time, some studies of LUAD have reported the ability to generate expression signatures or a single gene to group subjects according to their survival outcomes [34-38]. However, little has been reported for the inflammatory gene associated with cancer stemness to predict survival of patients with LUAD in a large dataset. The published gene TBX21 shows little overlap with the others as a significant predictor of outcome in LUAD. Thus, there is a strong possibility that this TBX21 gene could be used for diagnosis and prognosis prediction with large samples and adequacy of the different microarray platforms, contributing dramatically to the rational results.

In this study, we collected different datasets to generate a significant gene TBX21 to construct the prognostic model that could have potential clinical implementation. Significant emphasis was placed on the validation of this model by using a similar cutoff method to reduce technical variability 
in the other five GEO datasets. In the univariate and multivariate analysis, the significant factors represented the biology of the LUAD and associated clinical data. The prognostic power of TBX21 was analyzed with the log-rank test method and a significant result was obtained between patients with high-risk score and those with low-risk score. The OS rates of patients with high-risk score were significantly lower than those with low-risk score at 3 and 5 years, respectively. The median survival year was remarkably shorter for patients with high-risk score compared to those with low-risk score. A realistic assessment of the performance of this predictive model was executed in these datasets, and the HRs between high-risk and low-risk groups for all the datasets were more than 1.40. Furthermore, data stratification was performed among the three significant clinical factors (stage, age or gender) according to the risk level of TBX21 in LUAD patients from all GEO datasets. The rational results suggest that the prognostic model is independent of the other clinical factors. Especially in line with the research hypothesis, the predictive model of TBX21 could differentiate patients with poor survival and favorable survival within the same stage, age or gender stratum, which indicated the potential application of the prognostic model in predicting survival of patients with LUAD.

Importantly, it remains unknown whether the model has predictive power in LUAD patients from different clinical centers in China as this TBX21 predictive model was derived from the GEO datasets. Another limitation of our study is that the test set is not large enough to allow for a sensible assessment of generalizability for this predictive model. Datasets from other clinical departments and other countries are still necessary to validate its generalization ability. The validity of this gene model should be further confirmed in the prospective cohorts. Finally, our predictive model could potentially be applied to other NSCLC cancer types such as lung squamous cell carcinoma (LSCC). Our preliminary work in LSCC has shown equivalent results with those in LUAD (Additional file 3: Figure S2), but much more data for LSCC is needed to research their presence and clinical relevance in future.

Although more and more biomarkers were identified to predict the prognosis of LUAD during the past years [3941], functional study of biomarkers is still limited. Using the LUAD cell line A549, we discovered that TBX21 could promote sphere-forming capacity concomitant with upregulated expression of stemness biomarkers SOX2 and OCT4, suggesting that TBX21 increases the self-renewal of lung CSCs. In addition, TBX21 promotes the proportion of SP cells to display augmented drug resistance, another property associated with CSCs. Importantly, TBX21 induces cancer stemness biomarker (SOX2 and OCT4) alteration in LUAD cells and the mice model which is consistent with the acquisition of CSC maintenance. Overall, our studies provide the first evidence that TBX21 promotes cancer stemness which may contribute to LUAD growth, metastasis and recurrence.

Differentiation of $\mathrm{CD}_{4}^{+}$Th 1 and Th $2 \alpha \beta$ cells is tightly crossregulated, so that development of one subset is inhibited by cytokines produced by the other [29, 42] Considerable progress has been made in understanding the molecular mechanisms of this crossregulation. TBX21, an identified Th1-specific transcription factor selectively expressed in Th1 cells, plays a central role in Th1 development by activating Th1 genetic programs and repressing Th2 cytokine synthesis [30]. GATA3, in contrast, is a Th2-specific transcription factor selectively expressed in Th2 cells. It plays a major role in specifying the Th2 phenotype by promotion of Th2 cytokine secretion and inhibition of IFN- $\gamma$ production through repression of IL-12 signaling [29]. To our surprise, in our system our results showed that TBX21 and GATA3 have the same mRNA expression trends (Additional file 4: Figure S3). Moreover, our functional data underscore that TBX21 promoted IL-4 expression and IL-4 signaling is indispensable for TBX21-mediated cancer cell stemness. It is suggested that the reverse roles of genes may have the same contribution in tumor development.

\section{Conclusions}

In summary, we identified a novel model to predict survival in patients with LUAD by integrating currently available datasets. Based on the TBX21 expression value, we developed a risk score model dividing patients into a high-risk group and a low-risk group with significantly different prognosis. The robustness of this predictive model was successfully validated between the training dataset and the other five independent datasets for validation. Importantly, the prognostic power of this new model was derived from cancer stemness governed by the TBX21-IL-4 signaling pathway and was independent of the other clinical factors. Our research highlighted the potential roles of TBX21 as a new predictive biomarker and therapeutic target for LUAD patients.

\section{Additional files}

Additional file 1: Table S1. Presenting 1027 inflammation-related genes measured in this study. Loza et al. [20] identified associations between novel variants in inflammation-related genes and several common diseases, especially for some cancers. Genetic studies also provided evidence that some cancers were associated with inflammatory pathways which impact the risk of cancer initiation, progression and severity. (XLSX $17 \mathrm{~kb}$ )

Additional file 2: Figure S1. Showing stratification analysis of the TBX21 gene for stage, age and gender in integrated dataset including training dataset and five validation datasets. Kaplan-Meier survival curves between high-risk group and low-risk group for (A) stage I patients, (B) stage $\geq$ II patients, (C) young patients, (D) older patients, (E) female patients and (F) male patients. (TIFF $1760 \mathrm{~kb}$ )

Additional file 3: Figure S2. Showing prognostic model of TBX21 could be applied to patients with LSCC. (A) Heatmap shows genetic alterations 
analysis of TBX21 and five CSC biomarkers in LSCC patients $(n=504)$ with DFS or OS status from TCGA including amplification, deep deletion, mRNA upregulation, truncating mutation and missense mutation. (B) Synthetically altered ratios 5\%, 10\%, 6\%, 60\%, 6\% and 6\% for TBX21, NANOG, OCT4, SOX2, KLF4 and ALDH1A1 in LSCC patients, respectively. (C) Kaplan-Meier curves for overall survival between high-risk group and low-risk group defined by our predictive model based on TBX21 expression values in TCGA dataset. (D) DFS analysis of LSCC patients when stratified into high-risk group and low-risk group by TBX21 prognostic model in TCGA dataset. (TIFF $1239 \mathrm{~kb}$ )

Additional file 4: Figure S3. Showing TBX21, IL-4 and GATA3 gene expression in A549 cells. qPCR showed that mRNA expression of IL-4 and GATA3 was significantly upregulated with reconstitution of TBX21 expression in A549 cells $(P<0.001, t$-test method). (TIFF $201 \mathrm{~kb})$

\section{Abbreviations}

Cl: Confidence interval; CSC: Cancer stem cell; GEO: Gene Expression Omnibus; HR: Hazard ratio; IFN-ץ: Interferon gamma; IRG: Inflammationrelated gene; LSCC: Lung squamous cell carcinoma; LUAD: Lung adenocarcinoma; NSCLC: Nonsmall cell lung cancer; PCG: Protein-coding gene; TCGA: The Cancer Genome Atlas; TSS: Transcription start site

\section{Acknowledgements}

The authors thank Dr Rong Xiang (Department of Immunology, School of Medicine, Nankai University, China) for assistance in writing the paper and providing important advice.

\section{Funding}

This study was funded by China Postdoctoral Science Foundation (No. 179316, to SZ) and National High-tech Research and Development Projects (863) (No. 2015AA020106, to LW).

\section{Availability of data and materials}

The datasets during and/or analyzed during the current study are available from the corresponding author on reasonable request.

\section{Authors' contributions}

SZ collected GEO data samples, performed the biostatistics analysis and constituted the prediction model. WS validated the mechanism of the model with biological experiments. SZ and JY prepared and repaired the manuscript. LW designed the experiments and decided to publish the paper. All authors read and approved the final manuscript.

\section{Ethics approval and consent to participate}

The protocols in this study were reviewed and approved by the Ethical Committee and Institutional Review Board of the Cancer Hospital, Chinese Academy of Medical Sciences, and written informed consents were collected from each patient included in this research. This study did not involve the use of any animal tissue.

\section{Consent for publication}

All participants give their permission to publish this study.

\section{Competing interests}

The authors declare that they have no competing interests.

\section{Publisher's Note}

Springer Nature remains neutral with regard to jurisdictional claims in published maps and institutional affiliations.

\section{Author details}

'Department of Radiation Oncology, National Cancer Center/Cancer Hospital, Chinese Academy of Medical Sciences and Peking Union Medical College, Beijing 100021, China. ${ }^{2}$ Department of Pathology and Institute of Precision Medicine, Jining Medical University, Jining 272067, China. ${ }^{3}$ The School of Medicine, Nankai University, Tianjin 300071, China.
Received: 23 November 2017 Revised: 12 February 2018

Accepted: 26 February 2018 Published online: 03 April 2018

\section{References}

1. Chen W, Zheng R, Baade PD, Zhang S, Zeng H, Bray F, Jemal A, Yu XQ, He J. Cancer statistics in China, 2015. CA Cancer J Clin. 2016;66(2):115-32.

2. Hurt EM, Kawasaki BT, Klarmann GJ, Thomas SB, Farrar WL. CD44+ CD24prostate cells are early cancer progenitor/stem cells that provide a model for patients with poor prognosis. Br J Cancer. 2008;98(4):756-65.

3. Beier D, Wischhusen J, Dietmaier W, Hau P, Proescholdt M, Brawanski A, Bogdahn U, Beier CP. CD133 expression and cancer stem cells predict prognosis in highgrade oligodendroglial tumors. Brain Pathol. 2008;18(3):370-7.

4. Strauss GM. Adjuvant chemotherapy of lung cancer: methodologic issues and therapeutic advances. Hematol Oncol Clin North Am. 2005;19(2):263-81.

5. Crino L, Weder W, Van Meerbeeck J, Felip E, Group EGW. Early stage and locally advanced (non-metastatic) non-small-cell lung cancer: ESMO Clinical Practice Guidelines for diagnosis, treatment and follow-up. Ann Oncol. 2010; 21(Suppl 5):v103-15

6. Kajstura J, Rota M, Hall SR, Hosoda T, D'amario D, Sanada F, Zheng H, Ogórek B, Rondon-Clavo C, Ferreira-Martins J. Evidence for human lung stem cells. N Engl J Med. 2011;364(19):1795-806.

7. Botchkina G, Ojima I. Prostate and colon cancer stem cells as a target for anti-cancer drug development. Cancer Stem Cells Theories and Practice, InTech; 2011.

8. Lagadec C, Vlashi E, Della Donna L, Dekmezian C, Pajonk F. Radiation-induced reprogramming of breast cancer cells. Stem Cells. 2012;30(5):833-44.

9. Fabregat I, Malfettone A, Soukupova J. New insights into the crossroads between EMT and stemness in the context of cancer. J Clin Forensic Med. 2016;5(3):37.

10. Li Y, Wang L, Pappan L, Galliher-Beckley A, Shi J. IL-1 $\beta$ promotes stemness and invasiveness of colon cancer cells through Zeb1 activation. Mol Cancer. 2012;11(1):87

11. Zhao S, Bai N, Cui J, Xiang R, Li N. Prediction of survival of diffuse large Bcell lymphoma patients via the expression of three inflammatory genes. Cancer Med. 2016:5(8):1950-61.

12. Lazarevic V, Glimcher LH, Lord GM. T-bet: a bridge between innate and adaptive immunity. Nat Rev Immunol. 2013;13(11):777-89.

13. Miller SA, Weinmann AS. Molecular mechanisms by which T-bet regulates Thelper cell commitment. Immunol Rev. 2010;238(1):233-46.

14. Yu H, Yang J, Jiao S, Li Y, Zhang W, Wang J. T-box transcription factor 21 expression in breast cancer and its relationship with prognosis. Int J Clin Exp Pathol. 2014;7(10):6906.

15. Lin Z-W, Wu L-X, Xie Y, Ou X, Tian P-K, Liu X-P, Min J, Wang J, Chen R-F, Chen $Y$-J. The expression levels of transcription factors T-bet, GATA-3, RORyt and FOXP3 in peripheral blood lymphocyte (PBL) of patients with liver cancer and their significance. Int J Med Sci. 2015;12(1):7.

16. Zhao S, Fang S, Liu Y, Li X, Liao S, Chen J, Liu J, Zhao L, Li H, Zhou W. The long non-coding RNA NONHSAG026900 predicts prognosis as a favorable biomarker in patients with diffuse large B-cell lymphoma. Oncotarget. 2017; 8(21):34374-86

17. Zhao S, Dong X, Shen W, Ye Z, Xiang R. Machine learning-based classification of diffuse large B-cell lymphoma patients by eight gene expression profiles. Cancer Med. 2016:5(5):837-52.

18. Shen W, Du R, Li J, Luo X, Zhao S, Chang A, Zhou W, Gao R, Luo D, Wang J. TIFA suppresses hepatocellular carcinoma progression via MALT1-dependent andindependent signaling pathways. Signal Transduc Target Ther. 2016;1:16013.

19. Shen W, Chang A, Wang J, Zhou W, Gao R, Li J, Xu Y, Luo X, Xiang R, Luo N. TIFA, an inflammatory signaling adaptor, is tumor suppressive for liver cancer. Oncogene. 2015:4(10):e173.

20. Loza MJ, McCall CE, Li L, Isaacs WB, Xu J, Chang B-L. Assembly of inflammation-related genes for pathway-focused genetic analysis. PLoS One. 2007;2(10):e1035.

21. Lee S, Wottrich S, Bonavida B. Crosstalks between Raf-kinase inhibitor protein and cancer stem cell transcription factors (Oct4, KLF4, Sox2, Nanog). Tumor Biol. 2017;39(4):1010428317692253.

22. Liu Z, Zhang J, Kang H, Sun G, Wang B, Wang Y, Yang M. Significance of stem cell marker Nanog gene in the diagnosis and prognosis of lung cancer. Oncol Lett. 2016;12(4):2507-10.

23. Sedaghat S, Gheytanchi E, Asgari M, Roudi R, Keymoosi H, Madjd Z. Expression of cancer stem cell markers OCT4 and CD133 in transitional cell carcinomas. Appl Immunohistochem Mol Morphol. 2017;25(3):196-202. 
24. Lundberg IV, Edin S, Eklöf V, Öberg Å, Palmqvist R, Wikberg ML. SOX2 expression is associated with a cancer stem cell state and down-regulation of CDX2 in colorectal cancer. BMC Cancer. 2016;16(1):471.

25. Yu F, Li J, Chen H, Fu J, Ray S, Huang S, Zheng H, Ai W. Kruppel-like factor 4 (KLF4) is required for maintenance of breast cancer stem cells and for cell migration and invasion. Oncogene. 2011;30(18):2161-72.

26. Miyata T, Oyama T, Yoshimatsu T, Higa H, Kawano D, Sekimura A, Yamashita N, So T, Gotoh A. The clinical significance of cancer stem cell markers ALDH1A1 and CD133 in lung adenocarcinoma. Anticancer Res. 2017;37(5):2541-7.

27. Kim J-K, Jeon $\mathrm{H}-\mathrm{Y}$, Kim H. The molecular mechanisms underlying the therapeutic resistance of cancer stem cells. Arch Pharm Res. 2015:38(3):389-401.

28. Karamboulas C, Ailles L. Developmental signaling pathways in cancer stem cells of solid tumors. Biochim Biophys Acta. 2013;1830(2):2481-95.

29. Kanhere A, Hertweck A, Bhatia U, Gökmen MR, Perucha E, Jackson I, Lord GM, Jenner RG. T-bet and GATA3 orchestrate Th1 and Th2 differentiation through lineage-specific targeting of distal regulatory elements. Nat Commun. 2012;3:1268.

30. Yu F, Sharma S, Edwards J, Feigenbaum L, Zhu J. Dynamic expression of transcription factors T-bet and GATA-3 by regulatory $T$ cells maintains immunotolerance. Nat Immunol. 2015;16(2):197-206.

31. Chen Z, Fillmore CM, Hammerman PS, Kim CF, Wong KK. Non-small-cell lung cancers: a heterogeneous set of diseases. Nat Rev Cancer. 2014; 14(8):535-46.

32. Network CGAR. Comprehensive molecular profiling of lung adenocarcinoma. Nature. 2014:511(7511):543.

33. Cortés ÁA, Urquizu LC, Cubero JH. Adjuvant chemotherapy in non-small cell lung cancer: state-of-the-art. Transl Lung Cancer Res. 2015;4(2):191.

34. Li B, Cui Y, Diehn M, Li R. Development and validation of an individualized immune prognostic signature in early-stage nonsquamous non-small cell lung cancer. JAMA Oncol. 2017;3(11):1529-37.

35. Lin Y, LV Y, Liang R, Yuan C, Zhang J, He D, Zheng X, Zhang J. Four-miRNA signature as a prognostic tool for lung adenocarcinoma. Onco Targets Ther. 2018;11:29.

36. Jeong D, Ban S, Oh S, Lee SJ, Park SY, Koh YW. Prognostic significance of EDIL3 expression and correlation with mesenchymal phenotype and microvessel density in lung adenocarcinoma. Sci Rep. 2017;7(1):8649.

37. Peng F, Wang R, Zhang Y, Zhao Z, Zhou W, Chang Z, Liang H, Zhao W, Qi L, Guo Z. Differential expression analysis at the individual level reveals a IncRNA prognostic signature for lung adenocarcinoma. Mol Cancer. 2017;16(1):98.

38. Wu C, Zhang D. Identification of early-stage lung adenocarcinoma prognostic signatures based on statistical modeling. Cancer Biomark. 2017; 18(2):117-23.

39. Seike M, Kim C-H, Zou F, Noro R, Chiba M, Ishikawa A, Kunugi S, Kubota K, Gemma A. AXL and GAS6 co-expression in lung adenocarcinoma as a prognostic classifier. Oncol Rep. 2017;37(6):3261-9.

40. Meaney C, Zingone A, Brown D, Yu Y, Cao L, Ryan B. Identification of serum inflammatory markers as classifiers of lung cancer mortality for stage I adenocarcinoma. Oncotarget. 2017:8(25):40946-57.

41. Gao YL, Liu MR, Yang SX, Dong YJ, Tan XF. Prognostic significance of ACP5 expression in patients with lung adenocarcinoma. Clin Respir J. 2017:4(11):1-6.

42. Hosokawa H, Tanaka T, Endo Y, Kato M, Shinoda K, Suzuki A, Motohashi S, Matsumoto M, Nakayama Kl, Nakayama T. Akt1-mediated Gata3 phosphorylation controls the repression of IFN [gamma] in memory-type Th2 cells. Nat Commun. 2016;7:11289.

\section{Submit your next manuscript to BioMed Central and we will help you at every step:}

- We accept pre-submission inquiries

- Our selector tool helps you to find the most relevant journal

- We provide round the clock customer support

- Convenient online submission

- Thorough peer review

- Inclusion in PubMed and all major indexing services

- Maximum visibility for your research

Submit your manuscript at www.biomedcentral.com/submit

C Biomed Central 